\title{
La Psicología y su enseñanza en Argentina. Entrevista con Hugo Klappenbach
}

\author{
Education in Psychology in Argentina. Hugo Klappenbach's interview
}

\author{
Corina CALABresi* \\ Universidad Nacional de San Luis y Consejo \\ Nacional de Investigaciones Científicas \\ y Técnicas, Argentina \\ FERnANdo Polanco ** \\ Universidad Nacional de San Luis, \\ San Luis, Argentina
}

Para citar este artículo. Calabresi, C. \& Polanco, F. (2011). La Psicología y su enseñanza en Argentina. Entrevista con Hugo Klappenbach. Universitas Psychologica, 10 (2), 613-626.

\footnotetext{
Licenciada en Psicología y está realizando su doctorado en Psicología en la Universidad Nacional de San Luis. Becaria del CONICET e integrante del proyecto de investigación Incidencia de publicaciones periódicas y colecciones editoriales de Psicología en la formación del psicólogo en Argentina: 1954-1984. Email: cvcalabresi@unsl.edu.ar

** Licenciado en Psicología. Integrante del proyecto de investigación Incidencia de publicaciones periódicas y colecciones editoriales de Psicología en la formación del psicólogo en Argentina: 1954-1984. E-mail: fapiano2000@gmail.com
}

RESUMEN

Dialogamos con el reconocido investigador de la historia y enseñanza de la Psicología, Hugo Klappenbach, quien presenta una mirada crítica sobre la enseñanza de esta ciencia en Argentina, donde tiene como particularidad la impronta casi exclusiva del Psicoanálisis y la práctica clínica. Además, realiza una comparación con la formación profesional en otros países latinoamericanos, en norteamericanos y en europeos. Por último, señala las posibles proyecciones en la enseñanza y la investigación, tanto a nivel nacional como internacional.

Palabras clave autores

Psicología, enseñanza, Argentina, Klappenbach.

\section{A B S T R A C T}

We dialogue with Dr. Hugo Klappenbach, the well-known Latin-American researcher in the field of history of psychology as well as in the field of Psychology Education. Klappenbach gives a critical overview over the teaching of psychology in Argentina. From his perspective, psychology education in the whole country has been biased almost exclusively by Psychoanalysis and by clinical interests. Klappenbach compares such psychoanalytical perspective with teaching of psychology in other countries in Latin America, Europe or United States. Finally, he points out possible future directions related to psychology education both at national and international level.

Key words authors

Psychology, Teaching, Argentine, Klappenbach. 


\section{Introducción}

El Doctor Hugo Klappenbach es un prestigioso investigador en las áreas de la historia de la Psicología y la formación profesional del psicólogo. Ha realizado significativas contribuciones a la historia de la Psicología en Argentina y a nivel internacional, entre las que se encuentran estudios críticos de diferentes personajes prominentes como Aybar (Klappenbach, 2002), Alberini (Klappenbach, 1999a), Ardila (Klappenbach, 2003b), Krapf (Klappenbach, 2004a), Mira y López (Klappenbach, 1999b), Mouchet (Klappenbach, 1997), García de Onrubia (Klappenbach, 2008), Pichon Rivière (Klappenbach, 1987, 2007a), Piñero (Klappenbach, 1988, 1996), entre otros, además de una periodización de la Psicología argentina (Klappenbach, 2006). Además, ha realizado numerosos trabajos historiográficos de discusión epistemológica y teóricos sobre la práctica del historiador de la Psicología, inspirados principalmente en las visiones de Robert Watson, Kurt Dazinger, Helio Carpintero y Josef Brozek, algunos de los cuales llegarían a publicar en los Cuadernos Argentinos de Historia de la Piscología, revista editada por él.

Por otro lado, se ha especializado en temas como la enseñanza y práctica profesional del psicólogo, donde tuvo a su vez una participación activa tanto en el nivel profesional, en la Federación de Psicólogos de la República Argentina (FEPRA), como en el ámbito de la enseñanza, en las Asociación de Unidades Académicas de Psicología (AUAPsi) de universidades públicas argentinas.

Muchas de sus numerosas investigaciones fueron publicadas en las revistas más prestigiosas de Psicología, como la Revista Latinoamericana de Psicología o la Revista de Historia de la Psicología; y también en libros de alcance internacional, en los cuales fue invitado a participar, como por ejemplo, en el Handbook of International Psychology, editado por Stevens y Wedding (Klappenbach, 2004b) y en Advances in Psychology Research, editado por A. Columbus (Klappenbach, 2007b).

Actualmente, es Profesor Titular concursado de la cátedra de Historia de la Psicología en la Univer- sidad Nacional de San Luis e Investigador Principal del Consejo Nacional de Investigaciones Científicas y Técnicas (CONICET) de la República Argentina, donde dirige el proyecto de investigación, Incidencia de publicaciones periódicas y colecciones editoriales de Psicología en la formación del psicólogo en Argentina: 1954-1984.

En cuanto a las temáticas tratadas en la presente entrevista, Hugo Klappenbach nos da un panorama actual y crítico de la enseñanza de la Psicología en Argentina, que desde mediados de la década del 60 se ha caracterizado por su predominante orientación psicoanalítica, con especial énfasis en la práctica clínica (Klappenbach, 2000b, 2003a).

A su vez, se discuten las implicaciones de la enseñanza en universidades públicas y privadas, señalando las similitudes y diferencias entre ambos medios de profesionalización. Esto resulta interesante, tomando en cuenta que, a partir de este análisis, nuestro entrevistado se refiere a la formación de grado en las carreras de Psicología en Argentina respecto a otros países latinoamericanos, haciendo especial referencia a su posición respecto del Mercosur.

Por otro lado, hace un recorrido sobre la formación en Estados Unidos como también en Europa. Con base en ello, enfatiza el concepto de unificación de la Psicología, a partir de una organización epistémica e institucional, que se apoya en los planteos de Rubén Ardila (1993) y de Robert Sternberg y Elena Grigorenko (2001). Según Klappenbach, en el trabajo de investigación, una actitud científica consta de una amplia mirada desde diversas teorías psicológicas y, al mismo tiempo, el reconocimiento de los límites que ofrecen las diferentes ciencias y teorías de acuerdo al fenómeno estudiado. Por ello, se hace necesaria una verdadera interdisciplinariedad entre ciencias afines a la Psicología.

Su análisis sobre el futuro de la Psicología, tiene en cuenta el papel fundamental e indispensable que tendrá la investigación científica, tanto en su desarrollo como en la problemática que se plantea. Frente a esto, señala posibles alternativas para una mayor impronta de la investigación, dentro de la formación psicológica en la Argentina. 
Pregunta: Alonso y Gago (2009), en su último análisis cuantitativo de los psicólogos en Argentina, manifiestan que aquellas carreras pertenecientes a universidades privadas se han multiplicado. A su vez, el 35\% de los psicólogos egresados, corresponden a estas carreras. Con lo que se hace necesario preguntarse acerca de las semejanzas y diferencias entre la formación pública y privada. ¿Cuál es su opinión al respecto? y ¿Qué diferencias usted observa en la formación de estos dos ámbitos?

Hugo Klappenbach: Es muy difícil saberlo en detalle. En las universidades públicas, con todos los límites y problemas que debemos reconocer, hemos generado, no obstante, un conjunto de espacios públicos que permiten conocer y saber en detalle lo que estamos haciendo. Por ejemplo, consideremos el cuestionado Programa de Incentivos a la Investigación. Si alguien ingresa a la página de la Secretaría de Políticas Universitarias de Argentina y allí a la página del Programa de Incentivos, podrá encontrar una síntesis de todos los proyectos de investigación acreditados desde el año 1995 o 1996. Inclusive, encontrará los resultados de cada proyecto en cada año. Es decir, ha habido un esfuerzo fenomenal en volver públicas nuestras actividades de investigación; algo similar puede verificarse en la mayoría de las universidades públicas respecto a las actividades docentes. Todo esto ha generado más de una controversia dentro de las universidades públicas. No ha faltado quien vinculara esa actividad de publicidad, es decir, de hacer públicas nuestras actividades, con objetivos de control, característicos del Estado evaluador. Confieso que discrepo con ese tipo de planteos. Los docentes e investigadores de universidades públicas recibimos nuestro salario y nuestros subsidios de fondos públicos: icómo no va a poder saber la sociedad qué es lo que estamos haciendo?

Ahora bien, ise conoce algo análogo en las carreras de Psicología de las universidades privadas? No quisiera generalizar, pero me temo que no. De las universidades privadas conocemos relativamente poco. Es verdad que actualmente se puede ingresar a la página web de cualquier universidad privada en Internet y se puede encontrar el plan de estudios de Psicología. Son menos, en cambio, las que ofrecen el detalle de los docentes que se ocupan de cada asignatura. Y muchas menos las que ofrezcan una suerte de curriculum de los docentes o sobre sus actividades de investigación. Y ninguna, hasta donde yo he analizado, destaca el presupuesto destinado a la carrera, los fondos específicos para investigación, las aulas, laboratorios y espacios para practicum que poseen.

De todas maneras, creo que todo eso se va a ir modificando. En su momento, las carreras de Psicología de universidades privadas organizaron un espacio institucional análogo al de las universidades públicas AUAPsi (Asociación de Unidades Académicas de Psicología), la AUAPri, Asociación de Unidades Académicas de Psicología de Universidades Privadas, actualmente denominada UVAPsi, unidades de vinculación académica de Psicología.

Y en verdad, si las carreras de Psicología de universidades públicas y privadas pueden tener diferencias en cuestiones de financiación, de formas de gobierno y todas las diferencias políticas que conocemos, desde el punto de vista de la sociedad, hay un punto fundamental en común: en ambas se gradúan los futuros psicólogos y las futuras psicólogas que se desempeñarán profesionalmente. En ese sentido, cuando se analizan los contenidos de los dos congresos científicos que han realizado las carreras privadas nucleadas en UVAPsi -el último el año pasado-, se advierte un campo común de problemas académicos con el de las carreras de universidades públicas.

Análogamente, AUAPsi y UVAPsi presentaron un documento conjunto relacionado con la acreditación de las carreras de Psicología en el país. En esa dirección, más allá de sus diferencias, hay un núcleo de interés sobre la formación en Psicología que preocupa por igual a las carreras de Psicología de universidades públicas y de las universidades privadas.

Pregunta: Siguiendo con esta línea de análisis, en un artículo suyo "La globalización y la enseñanza de la Psicología en la Argentina" (Klappenbach, 2003a), usted analiza la enseñanza de la Psicología en Argentina respecto a los distintos momentos intervinientes en el proceso de diseño, evaluación 
e implementación efectiva de los planes de estudio, desde los tres niveles de concreción del curriculum, planteado por César Coll $(1992,1994)$. La pregunta es: icómo se aplica este análisis en la comparación entre carreras públicas y privadas?

Hugo Klappenbach: Sí, en efecto, César Coll destaca que el currículum o plan de estudios, no puede agotarse en el listado de materias o cursos. Eso constituye, apenas, lo que denomina el primer nivel del currículum, el llamado currículum explícito. El segundo nivel del currículo, está relacionado con las condiciones objetivas y materiales de la implementación del plan. Si en Argentina alguna carrera de Psicología propusiera la incorporación de dos o tres cursos de Psicología Experimental, desde el punto de vista del primer nivel de concreción del currículo, podría subrayarse la importancia que se le otorga a la Psicología Experimental. Sin embargo, si no se destina el presupuesto necesario para instalar laboratorios de Psicología Experimental y para contratar a docentes y auxiliares de laboratorio con la adecuada capacitación, esa importancia es meramente declarativa. Porque icómo se va a implementar la enseñanza de esa Psicología Experimental?, ¿en qué laboratorios?, icon qué personal? Porque, además, el personal de laboratorio no son solo los investigadores del mismo, sino también al personal auxiliar y a los técnicos con la capacitación, como diseñar o reparar instrumentos.

Y el tercer nivel de concreción del currículum, según César Coll, se relaciona con las prácticas efectivas de los docentes tanto en el aula como en los espacios curriculares que correspondan. Yo suelo usar el ejemplo de Psicología General porque creo que muchas veces en nuestro país es bien característico de que el primer nivel puede expresar algo, y en el tercer nivel, las cosas ir realmente por otro lado. Porque hay cursos de Psicología General que se confunden con Epistemología o con Historia de la Psicología o con Introducción al Psicoanálisis.

Si aplicamos esta conceptualización a lo que ocurre en Psicología en las universidades públicas y privadas, desde el primer nivel de concreción del currículo, no parece apreciarse excesivas diferencias. En todo caso, se aprecian diferencias entre las carreras de Psicología argentinas, por una parte, y otros modelos de enseñanza y entrenamiento en Psicología en otros lugares del mundo. Seguramente, no tendría sentido que comparáramos las carreras de Psicología en Argentina con las de Estados Unidos (sus major en Psicología), Inglaterra o Alemania. En cambio, creo que tiene sentido una comparación con la carrera en Chile, Colombia o México. La dilución de la Psicología en el Psicoanálisis atraviesa, en general, a todo el sistema, público y privado, aunque ya existen varias e interesantes excepciones en ambos sistemas. En general, la formación en Psicología se ha caracterizado por su insularidad respecto a la formación en Psicología en otras latitudes. Un ejemplo: ustedes saben bien que además de la Sociedad Interamericana de Psicología, existen otras instituciones internacionales de la Psicología, la Asociación Internacional de Psicología Aplicada y la IUPsyS (Unión Internacional de Psicología), que se organizó en 1951 y que reúne a asociaciones científicas de los diferentes países. La representación argentina la tiene la AACC (Asociación Argentina de Ciencias del Comportamiento). Sería interesante hacer una encuesta entre los psicólogos preguntando si conocen a IUPsyS. Estoy seguro que la enorme mayoría no la conoce. Me temo que tampoco conozcan demasiado a la SIP ni a la AACC. En cambio, la enorme mayoría de los graduados en Psicología podrían identificar sin inconvenientes y caracterizar con una enorme precisión a las instituciones internacionales de Psicoanálisis, desde la IPA hasta la Asociación Mundial de Psicoanálisis o Convergencia. De nuevo, creo que esto evidencia la insularidad local y la dilución de la Psicología en el Psicoanálisis.

Pregunta: En esta insularidad icómo queda la Psicología argentina, respecto a otros países latinoamericanos?

Hugo Klappenbach: Como ustedes saben, la Psicología latinoamericana existe desde, por lo menos, principios del siglo XX. Sin embargo, salvo el caso aislado del congreso en Montevideo en 1950, la Psicología latinoamericana toma conciencia de su identidad a finales de la década del sesenta. En ese sentido, la situación de la Psicología no difiere de lo que ocurría en otras Ciencias Sociales y, en 
realidad, de lo que ocurría en nuestras sociedades en sentido amplio. Rubén Ardila comienza a editar la Revista Latinoamericana de Psicología en 1969, cuando regresa de la Universidad de Nebraska con su doctorado finalizado. Esos años fueron muy significativos para toda América Latina, a partir de la expansión de movimientos contestatarios y revolucionarios, el impacto de la revolución cubana y de las luchas anticoloniales ejemplificadas en el caso de Argelia, el mayo francés y aun de los movimientos contestarios en los campus universitarios de Estados Unidos en rechazo de la intervención en Vietnam y del avance del movimiento de los derechos civiles en aquel país. En ese contexto, la Psicología latinoamericana, y así lo revelan las páginas de la Revista Latinoamericana de Psicología y también la Revista Interamericana de Psicología de la SIP, se caracterizó por un doble movimiento. Por una parte, un interés manifiesto por la aplicación de la Psicología a problemáticas sociales; por la otra, salvo en los casos de Argentina y Uruguay, por el desarrollo de una Psicología de corte científico y rigurosa en sus diseños metodológicas.

En este segundo movimiento, la Psicología argentina quedó aislada. Ustedes seguramente recordarán la amplia difusión del análisis experimental del comportamiento en toda América Latina, en un sentido progresista. Inclusive, en la experiencia de Los Horcones, en un sentido claramente revolucionario. En nuestro país, en cambio, salvo el caso de San Luis que ustedes han investigado ${ }^{1}$, era considerado de derecha.

Pregunta: Cristina Di Doménico y Alberto Vilanova (1999) realizaron una compilación, que reunió artículos de psicólogos de diversos países, donde se analiza la situación particular de los países que integran el Mercosur, Argentina, Brasil, Uruguay y Paraguay, dicho libro se denominó Formación de psicólogos en el Mercosur. ¿Cuál es su opinión con respecto a la formación en estos países, y en particu-

1 Se refiere al artículo denominado "Un precedente de la Psicología Conductual en la Universidad Nacional de San Luis" (Calabresi $\&$ Polanco, 2008), realizado por los entrevistadores. lar de las consecuencias de dicha integración sobre la formación en Argentina?

Hugo Klappenbach: Como ustedes señalan bien, Vilanova, Cristina di Doménico y su equipo analizaron con detalle la conformación, dentro del Mercosur del Comité Integrador de Psicólogos en el que participaban colegas de Brasil, Argentina, Uruguay y Paraguay. Se llegaron a un conjunto de acuerdos sumamente básicos sobre cuestiones de ejercicio profesional y de formación. En aquellos momentos, durante la década de los 90 , esos acuerdos, por elementales que puedan parecer, fueron de mucha importancia, porque, de alguna manera, evidenciaban que en el proceso de globalización y de integración de los países del Cono Sur, existían problemas comunes y, sobre todo, una agenda en común. No sé, en verdad, en que estado se encuentra hoy ni el Mercosur en general, ni el Comité Integrador de Psicólogos del Mercosur, en particular. Desde el gobierno de Alfonsín en adelante, al menos para Argentina, el Mercosur parecía ser una de las prioridades en materia de política exterior. Eso, para el caso de la Argentina me parece que fue de una importancia enorme, no sólo por cuestiones económicas y comerciales, sino sobre todo por cuestiones culturales. Durante muchas décadas en nuestro país, y muy especialmente en las zonas próximas al Río de la Plata, los argentinos nos considerábamos europeos en Sudamérica, actitud que inclusive generó cierto desdén hacia nuestros vecinos. Inclusive, como han analizado algunos historiadores, se produjo durante años una suerte de competencia con Brasil por el liderazgo en América del Sur. Las últimas tres décadas han demostrado dos cosas: la primera, que a partir de muy variados indicadores, en esa carrera competitiva con Brasil, nuestro vecino llevó la de ganar, ampliamente. En el caso de la Psicología, analicé hace un par de años atrás un aspecto sumamente parcial. Por ejemplo, en la base de datos PsycINFO se encuentran indizadas solamente cinco publicaciones de Psicología de Argentina. De Brasil, si no recuerdo mal, eran 26 o 27. La segunda, que para los intereses estratégicos de Argentina, en lugar de competir en una carrera con Brasil de resultados imprevisibles (recuerden por ejemplo que esa competencia incluyó también 
una peligrosa carrera armamentista) era preferible, en una actitud de mayor humildad, aceptar definitivamente el liderazgo regional de Brasil, y desde allí, promover instancias de integración, como el Mercosur, que posibilitaran un enriquecimiento mutuo.

Ahora bien, ihemos madurado lo suficiente en nuestro país como para aceptarnos a nosotros mismos como un socio menor de Brasil? No estoy del todo seguro. Además, en el caso de la Psicología, esa actitud no solo supone reconocer el mayor desarrollo académico de la Psicología del Brasil, evidenciado, por ejemplo, en la mejor visibilidad de sus publicaciones. También implica reconocer que, en materia de investigación de procesos básicos de la Psicología y aun en numerosos campos de aplicación, no solo Brasil nos ha superado, sino que también es posible verificar un desarrollo más sostenido que el nuestro también en países como Chile, Colombia, Venezuela, Puerto Rico, Cuba, Perú y, por supuesto, México. Ahora, podría argumentarse que tal vez esto pueda resultar así en el caso de la Psicología básica, pero que no ocurre lo mismo en la aplicación de la Psicología. Sin embargo, la presencia argentina en, por ejemplo, instituciones como la ALAPSO, la Asociación Latinoamericana de Psicología Social es muy reducida. De manera análoga, los servicios que ofrecemos a la comunidad desde nuestras carreras de Psicología también son bien reducidos.

Hace un año y medio atrás, tuvieron la amabilidad de invitarme como evaluador externo del proceso de autoevaluación de la Facultad de Psicología de la Universidad de la República en Uruguay. Muchos aspectos en la organización de la carrera de Psicología son similares a los de las carreras en Argentina. Sin embargo, dos cosas me llamaron gratamente la atención. La primera, el grado de autoconsciencia de las fortalezas y de las debilidades que tienen. Es decir, la Comisión de pares, que integramos con una colega de Brasil y un colega de Uruguay, en realidad tenía la finalidad de verificar los alcances de un proceso de autoevaluación que en la misma institución se había producido desde casi dos años atrás. Y, en efecto, pudimos verificarlo. Y la segunda, el desarrollo fenomenal de los servicios a la comunidad que ofrecía la Facultad de Psicolo- gía en Uruguay. El caso es por demás interesante, porque la carrera de Psicología del Uruguay también ha sido permeada por el Psicoanálisis, como tantas en nuestro país. Sin embargo, lo que se podía observar, era, ante todo, una práctica inspirada en el Psicoanálisis y fuertemente comprometida con la asistencia pública y con la promoción de la salud, en sentido amplio. En esa dirección, no creo que para el caso argentino, pueda servir el consuelo de que hemos aportado poco a la investigación básica, pero, en cambio, hemos realizado un aporte decisivo en términos de relevancia social. Lo cual no me sorprende. Como alguna vez me habrán escuchado sostener, estoy convencido de que la Psicología va a alcanzar un reconocimiento verdaderamente importante por parte de la sociedad, en la medida en que logremos afianzar dos principios que en modo alguno son incompatibles: la rigurosidad científica, por una parte, y la relevancia social, por la otra.

Por otro, en Brasil hay carreras de Psicología con una presencia importante del Psicoanálisis; pero yo tengo la impresión de que los psicoanalistas que están en las universidades en Brasil, ante todo, son académicos de la Psicología y, en segundo lugar, psicoanalistas. En Argentina fue exactamente al revés, primero fueron psicoanalistas y, me atrevería a afirmar, en segundo lugar, también. Es decir, se sacrificaron las características propias del espacio académico.

Pregunta: Respecto a su última afirmación de que en Argentina la formación es predominantemente psicoanalítica, y debido a que en numerosas publicaciones, tanto de su propia autoría (Klappenbach, 2000a, 2000b) como de otros autores (Alonso, 2000; Ardila, 1979; Vilanova, 1996, 2000), se ha llegado a la misma conclusión, nos preguntamos: ¿cómo es la formación en Psicología en otros lugares, como, por ejemplo, en Europa o Estados Unidos?

Hugo Klappenbach: Ante todo, me parece que en la mayoría de los países del mundo, el foco de la enseñanza no recae en macroteorías; más vale, está enfocada hacia problemas. ¿Qué quiero decir con esto? Por supuesto que cualquier investigación intenta dar cuenta de determinados objetos o fenómenos y procura transformar los datos sensibles en 
enunciados observacionales y, en algún momento dado, en enunciados teóricos, al mismo tiempo que las observaciones empíricas también se relacionan con presupuestos teóricos. La cuestión es icómo se llega a eso? En Argentina, es habitual partir de formulaciones tan generales que resulta muy difícil identificar los procesos que las generaron. Un ejemplo casi trivial y cotidiano: la afirmación de que lo afectivo tamiza los aspectos intelectuales. El problema no es si ese enunciado teórico es verdadero o falso; el problema es que la verificación de ese enunciado es bíblica o talmúdica; es verdadero porque así está escrito en los textos sagrados, en la obra de Freud o Lacan. La validez no reside en observaciones de investigaciones empíricas, sino en la fidelidad a los textos sagrados. Desde ya, el procedimiento no parece inadecuado para las Humanidades, pero parece poco pertinente para el campo científico. Por supuesto que se puede afirmar que, en términos demasiado amplios, la Psicología que se enseña en Europa, Estados Unidos y casi toda América Latina, es Psicología Cognitiva. Pero, de nuevo, no me refiero tanto a una matriz teórica como a los procesos que se estudian: los aspectos intelectuales y afectivos involucrados en los procesos de conocimiento. Es decir, Psicología Cognitiva pero no como una matriz teórica diferente del Conductismo o del Psicoanálisis. Psicología Cognitiva en tanto estudia los problemas inherentes al proceso de conocimiento. Robert Sternberg², al comienzo de este siglo, planteaba el proceso de unificación de la Psicología. Precisamente no planteaba esa unidad en términos teóricos o epistemológicos, más vale, sostenía que la organización epistémica y también la organización institucional, por ejemplo en los departamentos de Psicología de las universidades, debía estar cada vez más enfocada a ciertos problemas que era necesario estudiar como una unidad compleja. Por ejemplo, Sternberg se refería a los fenómenos de la memoria. ¿Quiénes estudian y se interesan por los procesos mnémicos? Por supuesto, es estudiada por los psicólogos cognitivos de laboratorio, interesados en procesos básicos, pero también los psicólogos sociales

2 Véanse Sternberg y Grigorenko (2001). interesados en la memoria e identidad colectivas. Y, finalmente, también por los psicólogos clínicos habituados a las perturbaciones de la memoria. Una tendencia que le preocupa a Sternberg es que, con mucha frecuencia, cada uno de estos grupos interesados en un aspecto o sector determinado de los procesos mnémicos, desconoce lo que investigan los otros. La unidad que reclama Sternberg, entonces, no es una unidad que provenga de una suerte de integración ecléctica de grandes matrices teóricas, epistemológicas o de grandes cosmovisiones, más vale, es la unidad de los problemas que nos vemos obligados a transformar en objetos acotados por necesidades de investigación. Si tomáramos como problema a, por ejemplo, la deserción escolar, es necesario el aporte de los que se ocupan de los procesos afectivos relacionados con el aprendizaje; por supuesto, de los que se ocupan de la Psicología Cultural capaz de analizar el valor cultural adjudicado a la escuela en amplios sectores de las sociedades contemporáneas, el aporte de los que se interesan por el desarrollo cognitivo y neurocognitivo de los niños. Es decir, puede apreciarse que la deserción escolar requiere de especialistas que provengan de la Psicología Educacional, Psicología Social, Psicología Cognitiva, Psicología Motivacional.

En la misma dirección, me parece que cuando enfocamos nuestra disciplina desde los problemas que enfrentan, las macroteorías y los grandes discursos teóricos se desvanecen o, por lo menos, pasan a un segundo plano. Por las dudas, porque en todo esto hay una gran confusión entre muchos de nuestros colegas y no quisiera que se malinterpretaran estas consideraciones, esto no significa afirmar que no que hay que preocuparse por las teorías. Lo que ocurre es que las teorías no pueden ocupar el lugar de un dogma de fe. Más aún, ihay alguna matriz dominante en esa corriente que los psicólogos norteamericanos denominan mainstream Psychology, es decir, la corriente principal de la Psicología? Solo en un sentido demasiado amplio. Porque la Psicología Cognitiva, si queremos otorgarle un carácter de macroteórica, es tan amplia, que caben allí enfoques tan diversos como los de Sternberg o Bruner o Cole o Wensch e, inclusive, enfoques diversos de aquellos que se denominan a sí mismos 
psicoterapeutas cognitivos. Inclusive, la Psicología Cognitiva, cada vez más, se ha venido ocupando de los aspectos motivacionales y afectivos y, por supuesto también, de los procesos inconscientes. Por supuesto, en un sentido diferente a la de aquella conocida imagen de Freud, la del ello como "un caldero de hirvientes estímulos". Pero hay cada vez más interés por los procesos inconscientes y cómo determinan comportamientos determinados. En ese sentido, entonces, la Psicología Cognitiva es realmente compleja y variada. La tendencia, que creo observar, es que las tres ciencias más cercanas de las que se nutre la Psicología, la Biología, la Antropología y la Sociología, son referencias cada vez más inevitables para la investigación psicológica y cada vez más se articulan y complementan entre sí y con la Psicología misma. En Argentina, todavía seguimos dominados por las grandes macroteorías. Es curioso, porque Freud sostenía que el Psicoanálisis no era una cosmovisión, no era una Weltanschauung. Coincido con esa apreciación; creo que lo más interesante que pueden aportar numerosas observaciones de los psicoanalistas, cuando se considera al Psicoanálisis como una Weltanschauung, esas posibilidades desaparecen y, a mi modo de ver, el Psicoanálisis languidece y se empobrece.

Pregunta: iY esto tiene que ver con una visión crítica en Psicología?

Hugo Klappenbach: Me parece que la cuestión pasa por reconocer cuál es el aporte interesante que una explicación teórica puede aportar a un fenómeno y, al mismo tiempo, tener la capacidad para reconocer los límites de tal explicación. En la imagen del foco de luz, poder aceptar que cuando dirijo el foco de luz de una determinada manera hacia un determinado fenómeno, se producen dos fenómenos dialécticamente complementarios. Por una parte, que efectivamente me permite alumbrar y visualizar ciertos rasgos del fenómeno analizado; por la otra, otros aspectos quedan en un cono de sombras y, por supuesto, otros totalmente obscurecidos. Me parece que la enseñanza de la Psicología que nos hemos acostumbrado a desarrollar en Argentina, inclusive en San Luis, donde hemos ampliado la oferta educativa a dos modelos teóricos, no nos ha permitido observar, en realidad, todas las zonas obscuras de las cuales no podemos dar cuenta. Y, por otro lado, tampoco nos ha permitido reconocer que, en general, el poder explicativo de las grandes teóricas psicológicas es sumamente elemental, casi de sentido común. ¿Qué quiero decir con esto? Ustedes saben que una de las características de las teorías científicas es su capacidad de generalizar determinados conocimientos y con ello de poder predecir ciertos comportamientos de partículas elementales, de cuerpos terrestres o celestes, de los organismos sobre los que se ha investigado. El grado de predicción de las teorías psicológicas es muy pobre, casi de sentido común. Por ejemplo, es posible predecir que si las figuras paternas, permanentemente maltratan y agreden físicamente a un hijo, el comportamiento de ese niño difícilmente sea el de una persona comprensiva ante las limitaciones y carencias afectivas de los otros. Ahora, ustedes notarán que es una predicción de sentido común, no requiero de ninguna investigación en Psicología para predecir esto. Por otro lado, también podría ocurrir un comportamiento totalmente contrario, en función de otras variables que no hemos considerado. En definitiva, carecemos de teorías que nos permitan predecir comportamientos más afinados. No sé del todo bien si esto se debe a la relativa juventud de las investigaciones psicológicas, o a que, quizás, el comportamiento de los individuos y los grupos sociales sea realmente muy difícil de predecir en los términos habituales de "dado este conjunto de características y fenómenos y dadas estas condiciones iniciales, se va a dar tal comportamiento". Esto muestra una debilidad de las teorías o, por lo menos, una limitación de las mismas.

Considero entonces que una perspectiva crítica en Psicología, implica reconocer, por una parte, todos los avances que la Psicología ha producido en poco más de un siglo. Por la otra, aceptar que existen limitaciones y zonas que siguen estando obscuras y, a tal fin, la Psicología requiere enfatizar todavía más la investigación. Investigar significa sencillamente buscar lo que no se conoce, implica una actitud individual de renuncia a las posiciones omnisapientes. Si yo sé todo, no preciso investigar. En cambio, cuando me planteo investigar un deter- 
minado tema, asumo, por una parte, todo lo que ya se conoce en publicaciones previas sobre ese tema o temas afines; por la otra, que desconozco muchas cosas de ese tema. Este proceso es clave para visualizar la necesidad de estudiar y conocer lo que se llama habitualmente "el estado del arte" o los "antecedentes del tema" que me interesa investigar. Cuando me planteo investigar un tema, parto de lo que han investigado generaciones anteriores, pero sobre todo mis contemporáneos sobre ese tema, y en ese proceso el manejo de las bases de datos y el manejo de los thesauros, para afinar la búsqueda en las bases de datos resultan fundamentales. ¿Cómo voy a poder realizar una investigación original, si desconozco las investigaciones análogas realizadas por mis pares?, ¿Y cómo voy a conocer adecuadamente esas investigaciones, si desconozco las bases de datos y por ende las publicaciones científicas donde se han publicado las nuevas investigaciones? Si yo no realizo nada de eso, mi investigación va a partir únicamente de lo que yo conozca por mi participación en una determinada institución o por los aportes de los clásicos. Y esto es muy limitado. Actualmente la base de datos más abarcativa en Psicología es PsycINFO, que incluye resúmenes de aproximadamente 2.500 revistas; en castellano existe PSICODOC, que indiza no menos de 600 revistas. Es decir, que solamente de las revistas indizadas en estas dos bases de datos, disponemos de más o menos 3.000 revistas. Si estimamos que cada revista pueda estar publicando, como promedio, tres números por año, estamos hablando de un mínimo de 9.000 números por año. Y si estimamos un promedio de diez artículos por número, estamos hablando de 90.000 artículos nuevos por año. Pues bien, en nuestro país, es bastante frecuente iniciar una investigación sin el recorrido inicial por las bases de datos que nos brindan al menos los resúmenes de esos 90.000 artículos. ¿Cómo puede llegar a plantearse, entonces, que se va a llevar a cabo un estudio original?

Es decir, creo que hay que poner mucho énfasis en la investigación, rigurosa y creativa, como toda buena investigación. Y, por supuesto, suficientemente acotada, de manera tal que la investigación pueda dar, efectivamente, resultados en los plazos establecidos. Y en la misma dirección, también considero que no existe a priori ninguna metodología especialmente recomendada para abordar un tema de estudio. Me interesa destacar esta cuestión, porque observamos toda una tendencia a justificar epistemológicamente el uso de los llamados métodos cuantitativos o cualitativos. Desde ya, no comparto esa perspectiva y creo que, en general, la combinación de ambos métodos es bastante frecuente. Hace apenas dos o tres años atrás, un grupo de suizos e ingleses comenzaron a publicar la Revista de Métodos Combinados de Investigación, el Journal of Mixed Methods Research. Me parece una respuesta saludable a tanta insistencia en ila inconmensurabilidad epistemológica o metafísica!

Pregunta: A nivel curricular, iqué sistemas de titulación existen? y iqué diferencias hay entre los programas de formación en Psicología en el mundo?

Hugo Klappenbach: Me parece que fuera de los países anglosajones, que tienen un sistema de titulaciones universitarias en tres niveles, el Bachelor, el Master y el Doctorado, que ahora puede ser el clásico Ph.D., es decir el Philosophical Doctor o el Psy.D., el doctorado en Psicología, en casi todos los países Europeos y en América Latina, tiene muchos puntos en común. En América Latina, por ejemplo, el modelo de la conferencia de Bogotá, de tres décadas atrás, todavía no se ha modificado en lo fundamental ${ }^{3}$. El título en Psicología es un título generalista. No existe una titulación de psicólogo clínico o psicólogo educacional, sino un título de psicólogo o licenciado o diplomado en Psicología, que habilita para cualquier actividad profesional. Por tratarse de un título generalista, se supone que por lo menos integra una formación básica general de procesos cognitivos, de procesos afectivos, de procesos psicosociales y, al menos, un paneo por los campos de la práctica canónicos: educacional, clínica, jurídica, social y comunitaria, y laboral. En Europa, desde los acuerdos de Bolonia, la tendencia parecía dirigirse a acortar las carreras de grado; algunas carreras de Psicología en universidades privadas

3 Véase Ardila (1978). 
se apresuraron a proponer carreras de cuatro años. Creo que es una tendencia que está en revisión para carreras de Psicología. La aprobación por parte de la Federación Europea de Asociaciones de Psicología del EuroPsy, aun cuando no se trate de una titulación universitaria sino de un certificado, ha puesto de manifiesto que las titulaciones en Psicología de cuatro años podrán existir, pero van a carecer de valor. Van a ser equivalentes a los Bachelor en Psicología que en realidad habilitan para muy pocas actividades. El certificado EuroPsy plantea un modelo de graduación en base a tres años de formación inicial, más dos de especialización y uno más de practicum. Es decir, lejos del modelo de cuatro años estamos en los hechos ante un modelo de seis años. Con lo cual, la formación óptima planteada en Psicología en Europa se asemeja mucho a la latinoamericana. En cualquier caso, si no queremos hablar de años sino de horas, la propuesta de formación nunca es inferior a 3.000 o 3.500 horas, lo cual implica, según los estándares internacionales, entre 6.000 y 7.000 horas de estudio. Es decir, estimando un año académico con 34 semanas y calculando un alumno full time, eso implica, otra vez, alrededor de 6 años.

Más allá de estas estimaciones, entre los expertos en acreditación curricular, al menos en Europa y en Latinoamérica, hay consenso en que la formación de un psicólogo o psicóloga en menos de cinco años no es lo más frecuente y, seguro, no es lo más deseable.

En cambio, un aspecto que sí es diferente en la formación europea en relación con la formación en Argentina, es que los contenidos relacionados con la formación en investigación se distribuyen por igual en el ciclo de formación básica y en el ciclo de aplicación o de formación profesional. Esto se corresponde con la concepción de que las actividades de investigación son medulares también para la vida profesional, de que es necesario entrenar en investigación al psicólogo clínico, educacional, laboral, y no solamente a aquellos interesados en dedicarse a la vida académica.

El debate sobre los contenidos y la cantidad de horas del plan de estudios, de todos modos, creo que hay que subordinarlo al debate sobre el perfil del graduado, es decir, que habilidades o competencias el graduado debe haber incorporado para obtener su titulación. Y si sigue estando vigente el modelo generalista, se desprende que hay competencias que no pueden dejar de enseñarse, aun cuanto ello implique la extensión de los estudios. Esto no es contradictorio con la idea de que el graduado tiene que seguir formándose y perfeccionando. En una comparación con el modelo anglosajón se podría plantear que allí el Bachelor tiene una duración menor, de 3 años; no es equivalente a nuestras licenciaturas. Pero, en efecto, las actividades a las que están habilitados con el Bachelor, aun si en el mismo se eligió la especialización en Psicología, el major como le llaman, son muy limitadas; actividades docentes secundarias, colaboración en la selección de personal o actividades de esas características. En los hechos, el verdadero título habilitante para ellos es el máster, con lo cual, de nuevo, estamos en cinco años. Inclusive, Estados Unidos es uno de los pocos países, para los cuales el doctorado es un título habilitante (en nuestra tradición y en la europea el doctorado es académico). Y allí, para la práctica de la psicoterapia se exige el nivel de doctorado, salvo en muy pocos estados que aceptan el título de máster. Adviertan entonces, que también allí, aun con un sistema diferente, ciertas competencias requieren seis o más años de formación.

Pregunta: Hasta ahora hemos hablado del pasado y del presente en la formación del psicólogo en Latinoamérica, Estados Unidos y Europa. En consecuencia y a partir de lo dicho, ¿qué posibles proyecciones piensa que tendrá la Psicología?

Hugo Klappenbach: Nunca es fácil hablar del futuro; corremos el riesgo de hacer futurología. A principios del nuevo siglo, Rubén Ardila publicó un libro muy interesante titulado precisamente $\mathrm{La}$ Psicología en el futuro ${ }^{4}$. El libro incluía entrevistas a personalidades relevantes y centrales de la Psicología de Europa, Estados Unidos, América Latina, Asia, África. Entre ellos a Robert Sternberg, John Adair, Marc Richelle, Philip Zimbardo, Ype Poortinga, Alan Kazdin, Ingrid Lunt, Helio Carpintero

4 Véase Ardila (2002). 
y muchos latinoamericanos: Rimoldi, Salazar, Julio Villegas, Díaz Guerrero, Dorna, de los que me acuerdo ahora.

Por supuesto, sería imposible sintetizar en pocas líneas la riqueza y diversidad de todas esas entrevistas. Sin embargo, el propio Rubén Ardila extrae algunas conclusiones que considero muy pertinentes. Creo recordar que una de las conclusiones es que la Psicología del futuro se apoyará cada vez más en la investigación científica; es decir, será cada vez más contrastable empíricamente y al mismo tiempo elaborará principios teóricos adecuados a los fenómenos analizados. Otra característica, que creo se enfatizaba, tenía que ver con acentuar cada vez más una perspectiva interdisciplinaria; no solo en el sentido de mejorar nuestros conocimientos sobre las bases biológicas, por una parte, y culturales y sociales, por la otra, de los fenómenos psicológicos, sino también de una mayor atención a cuestiones interdisciplinarias en la investigación aplicada y en la intervención profesional aplicada.

Por otro parte, una tendencia de la Psicología que posiblemente se vaya incrementando y creo que también destaca Rubén Ardila y muchos de sus entrevistados, es que la Psicología sea una herramienta útil a la sociedad. Las carreras universitarias son costosas para la sociedad, aun las carreras de Psicología. La sociedad, me parece, tiene bastante claro cuál es la utilidad social de un médico. Al menos en Argentina, me pregunto si la sociedad tiene claro cuál es la función de los psicólogos, fuera de la función del psicólogo clínico que está ampliamente difundida.

Pregunta: ¿Y respecto de la enseñanza de la Psicología en Argentina?

Hugo Klappenbach: En Argentina, en particular, creo que el futuro de la Psicología y el futuro de la enseñanza de la Psicología en particular, se enfrentan a un fenomenal desafío. De todas maneras, tampoco quisiera enfatizar únicamente los aspectos negativos. Al contrario, si comparamos el estado actual de las carreras de Psicología con la situación existente durante la dictadura militar, creo que la situación actual es, indudablemente, mejor. Con todos los problemas que cuando ustedes fueron estudiantes podían verificar diariamente en la formación, estoy seguro de que la formación de ustedes fue más diversa que la que yo tuve. En mi caso, con la excepción de la formación en Filosofía, que en verdad fue excelente, o en metodología, mi formación fue básicamente psicoanalítica y claramente orientada a la clínica psicoanalítica. No obstante, tampoco recorrimos, por ejemplo, a los psicoanalistas próximos a la Escuela de Frankfurt o a los culturalistas americanos, como Erich Fromm. Ustedes han recibido una formación más amplia, en general. Y no solamente porque han cursado aquí en San Luis, con sus dos enfoques teóricos, también es más amplia la formación en Córdoba, en La Plata, en Mar del Plata, en la UBA. Me parece, entonces, que el enorme desafío es poder profundizar todo esto, que esta tendencia se consolide y aun amplíe con el tiempo.

Durante dos o tres años fui el Coordinador de la Comisión de Carrera en mi universidad. Por tal motivo, me tocó presentar la carrera ante los nuevos ingresantes en el curso preparatorio. Recuerdo que trataba de plantearles a los y las estudiantes porqué razón habían elegido esta carrera. Para muchos, esa elección tenía que ver con que era una carrera que, por ejemplo, no exigía cursar Matemáticas y, en ese sentido, no parece ser una carrera difícil. Ustedes habrán advertido que en el imaginario de los estudiantes, Matemática o Física parecen carreras difíciles; en cambio Psicología no. Y trataba de razonar con los estudiantes que si, en efecto, la Psicología parece una carrera fácil, me temía que eso fuera un indicador de que no estamos haciendo del todo bien las cosas. Porque la Psicología exige buenos conocimientos en Biología, tanto como en Antropología y Sociología. Y, por supuesto, en Matemáticas y Lógica, como en cualquier otra disciplina científica. Es más, me gustaba enfatizarles a los y las estudiantes que Psicología, en cierto sentido, puede considerarse una de las carreras más difíciles que existen.

Creo que en el futuro se van a enfatizar las exigencias en la formación y se va a reconocer ampliamente la complejidad de la disciplina. Porque la Psicología, insisto siempre en esta cuestión, es al mismo tiempo una ciencia social y una ciencia natural, es ciencia y es profesión, es praxiología tanto como sensibilidad social y construcción teó- 
rica, exige conocimientos matemáticos tanto como responsabilidad ética en la investigación y en la actividad profesional.

Pregunta: ¿Considera que en Argentina finalmente se tomará conciencia de esa complejidad implicada en la enseñanza de la Psicología?

Hugo Klappenbach: Creo que sí. Además, me parece que las mayores exigencias van a provenir de frentes diferentes. Porque me parece que cuando esté suficientemente interiorizada esa complejidad, los primeros que nos van a exigir mucho más en nuestra función docente, van a ser los estudiantes. Las posibilidades que, cada vez más, ofrece Internet y el crecimiento sostenido de publicaciones científicas en texto completo y gratuito en Internet, ha acelerado un fenómeno que en Argentina Alberto Vilanova había previsto. No va a estar demasiado lejano el día en que los y las estudiantes hayan navegado intensamente por Internet y lleguen a los cursos sabiendo contenidos y diseños metodológicos bien actualizados, más actualizados que los nuestros, sobre todo si los docentes no advertimos que la actualización es hoy tarea cotidiana.

Por otro lado, los graduados y las graduadas en Psicología son muchas veces demandados por personas o grupos sociales que sufren. Esto nos plantea una serie de advertencias no solo sobre nuestras responsabilidades éticas, sino también sobre la actualización de nuestros conocimientos. De la misma manera, cuando realizamos investigaciones. Y no pensemos en investigaciones de laboratorio. En mi mismo campo de investigación, la historia de la Psicología, hace pocos años atrás no era una preocupación muy extendida la cuestión de la confidencialidad de los datos suministrados por un testimoniante clave. Hoy es todo un tópico que discuten historiadores y antropólogos junto con especialistas en ética.

Entonces, me parece que desde la sociedad misma, van a incrementarse las exigencias de regulaciones y controles éticos cada vez más rigurosos, al mismo tiempo que cada vez más participativos en su generación. No me refiero tanto a exigencias externas a la comunidad de pares. Al contrario, me parece que lo más necesario es que los psicólogos y psicólogas participen en las decisiones que regulen la investigación y que regulen la profesión.

Ahora bien, me parece que esas responsabilidades también se van a acrecentar para la profesión docente. Como docentes tenemos una enorme responsabilidad social en el perfil de nuestros graduados y nuestras graduadas. Además, para aquellos docentes en universidades públicas, tenemos una responsabilidad extra, porque nuestros salarios los abona el Estado, que es como decir que los abona la sociedad en su conjunto, que es la que sostiene las cargas fiscales. En ese sentido, tenemos un doble compromiso ético y social como docentes de carreras públicas de Psicología.

Tengo la impresión de que todas estas exigencias se vienen a pasos agigantados. Ya hemos ingresado, y creo que no siempre somos del todo concientes de ello, en una etapa que exige una sólida investigación sobre las bases biológicas del comportamiento, la subjetividad y los procesos mentales; simultáneamente, una igualmente sólida investigación sobre los componentes sociales, antropológicos y culturales del comportamiento, la subjetividad y los procesos mentales. De la mano de ellos y para las grandes problemáticas contemporáneas, desde la deserción escolar hasta la violencia, la drogodependencia o las distintas formas de explotación humana, cada vez más se nos va exigir un entrenamiento para la investigación y la intervención interdisciplinarias. Es posible que un psicólogo o psicóloga no puedan solucionar por sí solos esos problemas; en cambio, es posible la participación de un psicólogo o una psicóloga en equipos interdisciplinarios capaces de abordar el problema en toda su complejidad. Me parece que en el futuro, y un futuro bien cercano, imagino cada vez más a los psicólogos y psicólogas involucrados en la investigación y en el abordaje profesional de estas grandes problemáticas.

Pregunta: Tomando sus últimas palabras y ya para finalizar, iqué se puede hacer para fortalecer la investigación en la Psicología?

Hugo Klappenbach: Ante todo, diría que es muy difícil una respuesta sencilla. En primer lugar, considero que la investigación se va a fortalecer, si en nuestras carreras existen docentes que se dediquen 
habitualmente a la investigación. Desde ya, eso no quiere decir que tengan que estar en la carrera del investigador del CONICET, ni siquiera en el Programa de Incentivos, más vale, me refiero a que la investigación forme parte de sus hábitos cotidianos. En segundo lugar, hay universidades que tienen organizada una carrera del investigador, como la Universidad Nacional de Rosario. Habría que preguntarse por la efectividad de ese sistema, pero en cualquier caso no dejar de ser interesante. En tercer lugar, me parece que si un número importante de docentes investigan, los estudiantes van a percibir que investigar ya no es más lo otro, lo extraño, sino algo habitual en las actividades de formación. En cuarto lugar, y esto me parece fundamental, me parece que muchas veces transmitimos la sensación de que investigar es una tarea muy difícil o que requiere de habilidades especiales que no se encuentran en el común de la personas. Desde ya, esto no es así; para investigar solo se necesita pasión por el conocimiento, inquietudes y ganas de aprender. El entrenamiento en las variadísimas técnicas de investigación se puede aprender rápidamente, si partimos de aquella vocación o pasión por el conocimiento. En quinto lugar, y para el caso de nuestra carrera de Psicología en San Luis, una medida muy sencilla para estimular la investigación, sería reglamentar las pasantías en investigación como parte de la carrera, es decir, como una actividad equivalente a un curso electivo. Al lado de todo esto, algo que es fundamental para incrementar la investigación, es incrementar la lectura de bibliografía actualizada. Yo suelo decir que cuando leemos buenas investigaciones, no solo aprendemos sobre el tema de la investigación, sino sobre diferentes tipos de diseños para la recolección o construcción de los datos.

Creo que, efectivamente, al frecuentar publicaciones de otros investigadores, aprendemos sobre lo que ellos están investigando, aprendemos los diseños de investigación que utilizaron, aprendemos la modalidad del recorte de objetos que ellos realizaron, y todo eso va ayudando en la formación constante de un investigador. En el campo en que yo investigo, creo que les comentaba el otro día lo fascinado que había estado con el libro de Alejandro Blanco sobre Gino Germani. Más aún, a pesar de que explícitamente su objeto de estudio era la obra de Germani y la conformación de la Sociología científica en Argentina, me atrevo a decir que es uno de los mejores libros sobre historia de la Psicología en Argentina que he leído en los últimos tiempos. Pero no solo por lo que el libro investiga, por la relación de Germani con Butelman y su trabajo en la editorial Paidós; también por lo que sugiere. Por ejemplo, el libro examina la Sociología de cáte$d r a$, anterior a Germani. Hay múltiples analogías y también muchísimas diferencias para comparar con el desarrollo de la Psicología anterior a la carrera, especialmente en el período peronista, que el libro no examina. Pero leyendo ese libro, rápidamente es posible pensar: "icaramba!, lo que yo estoy leyendo es de interés aplicarlo, en el mismo período, para otra disciplina afín"

Creo que uno de los problemas serios en la investigación psicológica en Argentina, es que el Programa de Incentivos obligó a numerosos docentes que durante años no habían frecuentado publicaciones periódicas ni habían publicado artículos, a incorporarse a un sistema que era totalmente desconocido y externo. En efecto, si no es habitual frecuentar las publicaciones periódicas, es muy difícil que esté interesado en publicar en ellas. En fin, posiblemente sea un círculo vicioso.

\section{Referencias}

Alonso, M. \& Gago, P. (2009). Psicólogos en Argentina, actualización cuantitativa 2008. En Memorias de las XVI Jornadas de Investigación y Quinto Encuentro de Investigadores en Psicología del Mercosur (Vol. 3, p. 464). Buenos Aires: Facultad de Psicología, Universidad de Buenos Aires.

Ardila, R. (Ed.). (1978). La profesión del psicólogo. México: Trillas.

Ardila, R. (1979). La Psicología en Argentina: pasado, presente y futuro. Revista Latinoamericana de Psicología, 2 (1), 77-91.

Ardila, R. (1993). Sintesis experimental del comportamiento (2⿳亠丷⿵冂丶 ed.). Bogotá: Planeta.

5 Véase Blanco (2006). 
Ardila, R. (2002). La Psicología en el futuro. Madrid: Pirámide.

Blanco, A. (2006). Razón y modernidad. Gino Germani y la sociología en la Argentina. Buenos Aires: Siglo XXI.

Calabresi, C. \& Polanco, F. (2008). Un precedente de Psicología conductual en la Universidad Nacional de San Luis (Argentina). Memorandum, 15, 52-60.

Coll, C. (1992). Psicología y curriculum (3ª reimpresión). Barcelona: Paidós Ibérica.

Coll, C. (1994). Posibilidades críticas en el desarrollo de la reforma curricular española. Substratum, 2 (5), 125-150.

Di Doménico, C. \& Vilanova, A. (1999). Formación de psicólogos en el Mercosur. Mar del Plata: Universidad Nacional de Mar del Plata.

Klappenbach, H. (1987). Bibliografía de Pichon Rivière. Actualidad Psicológica, 133, 22-23.

Klappenbach, H. (1988). Horacio G. Piñero y la Psicología Experimental argentina. Boletín Argentino de Psicología, 2, 12-15.

Klappenbach, H. (1996). Prólogo a la Psicología Experimental en la República Argentina de Horacio Piñero. Cuadernos Argentinos de Historia de la Psicología, $2(1 / 2), 239-268$.

Klappenbach, H. (1997). Enrique Mouchet, el Psicoanálisis y la Psicología vital. Cuadernos Argentinos de Historia de la Psicología, 3 (1/2), 141-158.

Klappenbach, H. (1999a). La recepción orteguiana, Alberini y la renovación de la Psicología argentina a partir de los veinte. Revista de Historia de la Psicología, 20 (1), 87-95.

Klappenbach, H. (1999b). Obstáculos de Mira y López en la Argentina. Acta Psiquiátrica y Psicológica de América Latina, 45 (3), 274-280.

Klappenbach, H. (2000a). El Psicoanálisis en los debates sobre el rol del psicólogo. Argentina, 1960-1975. Revista Universitaria de Psicoanálisis, 2, 191-227.

Klappenbach, H. (2000b). El titulo profesional de psicólogo en Argentina antecedentes históricos y situación actual. Revista Latinoamericana de Psicología, 32 (3), 419-446.

Klappenbach, H. (2002). Benjamín Aybar y el desarrollo de la psicotecnia y la orientación profesional. En N. Horenstein, L. Minhot \& H. Severgnini (Eds.), Epistemología e Historia de la Ciencia. Selección de trabajos de las XII Jornadas (Vol. 8, pp. 183-189).
Córdoba: Centro de Investigaciones de la Facultad de Filosofía y Humanidades, Universidad Nacional de Córdoba.

Klappenbach, H. (2003a). La globalización y la enseñanza de la Psicología. Psicologia em Estudo, 8 (2), 3-18.

Klappenbach, H. (2003b). Rubén Ardila y la epistemología de la Psicología. En L. Flórez Alarcón (Ed.), El legado de Rubén Ardila. Psicología: de la biología a la cultura (pp. 45-81). Bogotá: Universidad Nacional de Colombia.

Klappenbach, H. (2004a). Eduardo Krapf (1901-1963): Primer Presidente de la Sociedad Interamericana de Psicología. Revista Interamericana de Psicologíal Interamerican Journal of Psychology, 38 (2), 361-367.

Klappenbach, H. (2004b). Psychology in Argentina. In M. Stevens \& D. Wedding (Eds.), Handbook of International Psychology (pp. 129-150). New York: Brunner-Routledge.

Klappenbach, H. (2006). Periodización de la Psicología en Argentina. Revista de Historia de la Psicología, 27 (1), 109-164.

Klappenbach, H. (2007a). A formação de psicólogos na Argentina: a psicologia social de Pichon Rivière. Em A. M. Jacó-Vilela \& L. Sato (Eds.), Diálogos em Psicologia Social (pp. 159-183). Porto Alegre: Editora Evangraf/Abrapso Sul.

Klappenbach, H. (2007b). Professional psychologist degree in Argentina: From the beginnings to nowadays. In A. Columbus (Ed.), Advances in Psychology Research (Vol. 38, pp. 1-32). Hauppauge, NY, US: Nova Science Publishers.

Klappenbach, H. (2008). Luis Felipe García de Onrubia: de la crítica al análisis factorial, a la consideración de Sartre y Freud. Temas de Historia de la Psiquiatría Argentina, 25, 24-30.

Sternberg, R. \& Grigorenko, E. (2001). Unified Psychology. American Psychologist, 56 (12), 1069-1079.

Vilanova, A. (1996). Enseñanza de la Psicología: historia y problemas fundamentales. Cuadernos Argentinos de Historia de la Psicología, 2 (1/2), 199-210.

Vilanova, A. (2000). La formación académica del psicólogo en el mundo y en el país. En O. Calo \& A. Hermosilla (Eds.), Psicología, ética y profesión: aportes deontológicos para la integración de los psicólogos del Mercosur (pp. 107-118). Mar del Plata: Universidad Nacional de Mar del Plata. 